\title{
ALMA observations of the Red Rectangle, a preliminary analysis ${ }^{\star}$
}

\author{
V. Bujarrabal ${ }^{1}$, A. Castro-Carrizo ${ }^{2}$, J. Alcolea ${ }^{3}$, H. Van Winckel $^{4}$, C. Sánchez Contreras ${ }^{5}$, M. Santander-García ${ }^{3,6}$, \\ R. Neri ${ }^{2}$, and R. Lucas ${ }^{7}$ \\ 1 Observatorio Astronómico Nacional (OAN-IGN), Apartado 112, 28803 Alcalá de Henares, Spain \\ e-mail: v.bujarrabal@oan.es \\ 2 Institut de Radioastronomie Millimétrique, 300 rue de la Piscine, 38406 Saint Martin d'Hères, France \\ 3 Observatorio Astronómico Nacional (OAN-IGN), C/ Alfonso XII, 3, 28014 Madrid, Spain \\ 4 Instituut voor Sterrenkunde, K.U. Leuven, Celestijnenlaan 200B, 3001 Leuven, Belgium \\ 5 Centro de Astrobiología (CSIC-INTA), ESAC Campus, 28691 Villanueva de la Cañada, Madrid, Spain \\ 6 Centro de Astrobiología (CSIC-INTA), Ctra. M-108, km. 4, 28850 Torrejón de Ardoz, Madrid, Spain \\ 7 UJF-Grenoble1/CNRS-INSU, Inst. de Planétologie et d'Astrophysique de Grenoble (IPAG) UMR 5274, 38041 Grenoble, France
}

Received 9 July 2013 / Accepted 19 July 2013

\section{ABSTRACT}

\begin{abstract}
Aims. We aim to study equatorial disks in rotation and axial outflows in post-AGB objects so as to disclose the formation and shaping mechanisms in planetary nebulae. So far, both disks and outflows have not been observed simultaneously.

Methods. We obtained high-quality ALMA observations of ${ }^{12} \mathrm{CO}$ and ${ }^{13} \mathrm{CO} J=3-2$ and ${ }^{12} \mathrm{CO} J=6-5$ line emission in the Red Rectangle, the only post-AGB/protoplanetary object for which a disk in rotation has been mapped.

Results. These observations provide an unprecedented description of the complex structure of this source. Together with an equatorial disk in rotation, we find a low-velocity outflow that more or less occupies the region situated between the disk and the optical X-shaped nebula. From our observations and preliminary modeling of the data, we confirm the previously known properties of the disk and obtain a first description of the structure, dynamics, and physical conditions of the outflow.
\end{abstract}

Key words. stars: AGB and post-AGB - circumstellar matter - radio lines: stars - planetary nebulae: individual: Red Rectangle

\section{Introduction}

Many protoplanetary nebulae (PPNe) show very massive $\left(\sim 0.1 M_{\odot}\right)$ and fast $\left(30-200 \mathrm{~km} \mathrm{~s}^{-1}\right)$ bipolar outflows, which are thought to be crucial in the formation of planetary nebulae; see, e.g., Bujarrabal et al. (2001) and Balick \& Frank (2004). These outflows carry too much linear momentum to be powered by momentum transfer from stellar photons, and the presence of disks rotating around the central stars and reaccretion from them are often postulated for explaining the nebular dynamics (e.g., Soker 2001; Frank \& Blackman 2004). However, only one of these putative disks has been identified well to date, which was by means of interferometric maps of ${ }^{12} \mathrm{CO} J=1-0$ and $J=2-1$ emission in the Red Rectangle (Bujarrabal et al. 2005). The Red Rectangle is a well studied PPN and, curiously, one of the post-asymptotic giant branch (AGB) objects that do not show massive and very fast outflows. It consists of the equatorial rotating disk plus a spectacular axisymmetric nebula seen in the visible, surrounding a double stellar system (Men'shchikov et al. 2002; Cohen et al. 2004). It also shows properties, such as a near-infrared (NIR) excess that is indicative of hot dust kept close to the stellar system, which suggest the presence of a very compact disk (e.g. Van Winckel 2003).

Recent single-dish observations of ${ }^{12} \mathrm{CO}$ and ${ }^{13} \mathrm{CO} \mathrm{mm}$ wave emission in a sample of similar post-AGB stars (close binary stars with low-mass nebulae and indications of compact disks) systematically yielded characteristic line profiles,

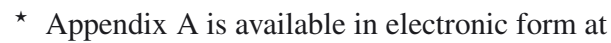
http: //WwW . aanda.org with a prominent single or double peak and moderate-velocity wings, which are strikingly similar to those of the Red Rectangle (Bujarrabal et al. 2013, Paper II). Profiles of this kind are also found in disks around young stars, and they have been proven to be very reliable indicators of rotating disks, both on theoretical and observational grounds.

Remarkably, gas in slow expansion has also been detected in some of these post-AGB objects. CO maps of 89 Her show an extended component expanding at about $5 \mathrm{~km} \mathrm{~s}^{-1}$, whose emission dominates the line wings (Bujarrabal et al. 2007); this source also shows a barely resolved central condensation that is probably in rotation. From careful modeling of Herschel observations of high- $J$ CO lines in the Red Rectangle, Bujarrabal \& Alcolea (2013, Paper I) deduced that a (probably bipolar) component in expansion should contribute significantly to the high- $J$ line wings in this source. But the spatial resolution of those observations was poor and the existence of such a component remained very uncertain. Indications of molecule-rich outflows were also found for other similar sources, in which the disk-like CO profiles show relatively strong line wings (Paper II).

All these nebulae, including the Red Rectangle, show low values of the total mass $\left(\lessgtr 10^{-2} M_{\odot}\right)$ and expansion velocity (5-10 $\mathrm{km} \mathrm{s}^{-1}$ ), unlike for the massive PPNe mentioned before, and may represent a different post-AGB evolutionary regime. The Red Rectangle is the prototype of this important class of objects and the best target for studying the simultaneous appearance of rotating disks and bipolar outflows, which is a basic input for understanding the formation and shaping of planetary nebulae. 


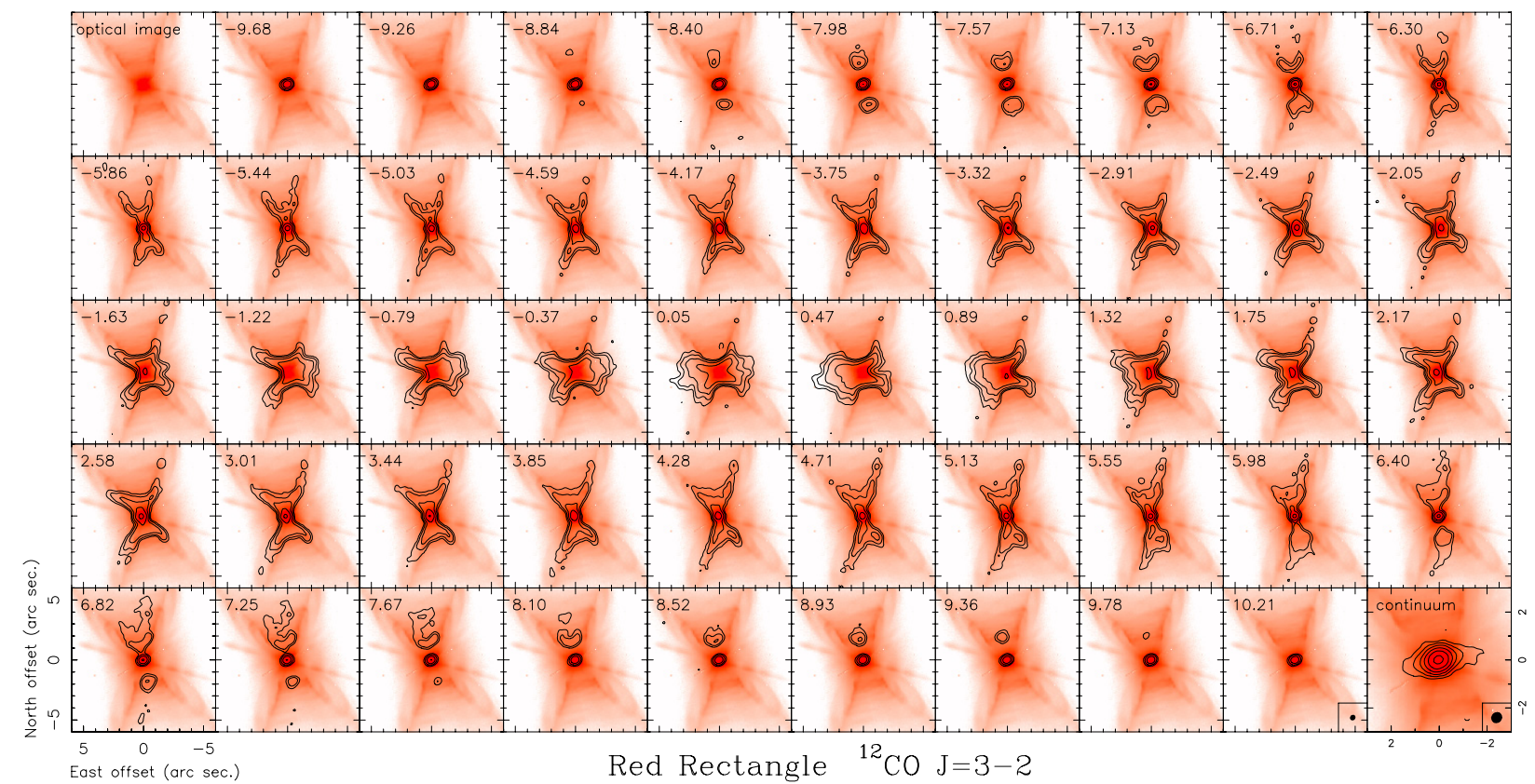

Fig. 1. ALMA ${ }^{12} \mathrm{CO} J=3-2$ observations of the Red Rectangle. The maps are centered at J2000 coordinates 06:19:58.205, $-10: 38: 15.00$. The LSR velocity is indicated in the upper-left corners. The contour spacing is logarithmic: $-0.03,0.02,0.06,0.18,0.54$, and $1.62 \mathrm{Jy} / \mathrm{beam}$ (equivalent to $0.67,2.0,6.1,18.2$, and $54.5 \mathrm{~K}$ ). The HST optical image is also displayed, and the last panel shows the $0.85 \mathrm{~mm}$ continuum image zoomed by a factor 2 and with contours $\pm 0.0015,0.0045,0.0135,0.0405,0.1215$, and $0.3645 \mathrm{Jy} / \mathrm{beam}$. The inserts in the two last panels show the beam width.

\section{Observations}

We present ALMA observations of the Red Rectangle, using receiver bands 7, to map the ${ }^{12} \mathrm{CO}$ and ${ }^{13} \mathrm{CO} J=3-2$ transitions, and 9, to map the CO $J=6-5$ transition. The backends were set to achieve a spectral resolution of $0.21 \mathrm{~km} \mathrm{~s}^{-1}$. Band 7 observations were performed in October 2012 and those of band 9 in November; the data were delivered in December 2012. During the observations, the array consisted of 21 to 24 antennas distributed in a configuration that extended up to $380 \mathrm{~m}$. RF and flux calibrators were observed in each run, and two phase calibrators were observed alternatively with the source. The delivered calibrated data were significantly improved with a better adapted calibration strategy. See more details in Appendix A.

The image deconvolution was made by using robust weighting (with a threshold of 1) for band 7 and natural weighting for band 9. The resulting brightness $\mathrm{rms}$ is $5 \mathrm{mJy} / \mathrm{beam}$ in the $\mathrm{CO}$ $J=3-2$ channel maps, for a spectral resolution of $0.42 \mathrm{~km} \mathrm{~s}^{-1}$ and a synthetic beam with a HPBW of $0{ }^{\prime} 48 \times 00^{\prime} 44$. The rms becomes $\sim 7 \mathrm{mJy} /$ beam in the channel maps with the brightest emission. For ${ }^{12} \mathrm{CO} J=6-5$ maps the $\mathrm{rms}$ is $\sim 30 \mathrm{mJy} / \mathrm{beam}$ with a synthetic beam of $0 ! 31 \times 0{ }^{\prime} 25$ and $0.42 \mathrm{~km} \mathrm{~s}^{-1}$ resolution. For the continuum maps, a brightness rms of $3 \mathrm{mJy} / \mathrm{beam}$ is obtained in band 7 and of $8 \mathrm{mJy} /$ beam in band 9 . The continuum emission extends in total $\sim 3^{\prime \prime}$ in the direction of the equatorial disk. The integrated continuum flux varies, because of the source spectral index, in the ranges $0.59-0.66$ and 3.2-3.6 Jy for bands 7 and 9, respectively. Calibration was made with the CASA software package, while we used GILDAS for the imaging and data analysis. An optical image was obtained from the HST archive and used for comparison with the $\mathrm{CO}$ data; see details in Appendix A.

\section{Results and simple modeling of our ALMA maps}

Our maps (Figs. 1-3) show the complex structure of the Red Rectangle. The already known rotating disk in the nebula equator (previously mapped in ${ }^{12} \mathrm{CO} J=1-0$ and $J=2-1$ with lower quality by Bujarrabal et al. 2005) is accurately described in our maps. Together with this disk, an X-shaped structure delineating the lobes of the optical nebula is mapped in molecular emission for the first time. This component must be in expansion, because their emission is detected at too high velocities and too large distances to the center. (The gravitational attraction is given by the rotation in the disk, which shows high velocities only very close to the center, as expected for a Keplerian or Keplerian-like law; see Bujarrabal et al. 2005.)

The presence of CO-emitting outflows in the Red Rectangle was suggested in Paper I from careful modeling of $\mathrm{mm}$-wave and FIR CO lines, but only indirect indications of the outflow were provided and qualitative ideas on its spatial distribution and physical conditions could be deduced. Our results clearly confirm that prediction. The emission from the X-shaped component is indeed dominant in the ${ }^{12} \mathrm{CO} J=6-5$ line, but is rather weak in the ${ }^{13} \mathrm{CO} J=3-2$ one.

Both ${ }^{12} \mathrm{CO}$ and ${ }^{13} \mathrm{CO} J=3-2$ show a similar peak brightness in the disk, with a remarkably high value that is not much lower than the expected kinetic temperatures ( 100 K, Paper I), showing that, in the central regions, these lines are mostly thermalized and optically thick. The peak brightness of ${ }^{12} \mathrm{CO} J=$ 6-5 is also comparable, but its intensity significantly decreases toward the end of the disk, indicating that its excitation is relatively low in the outer disk. The outflowing component is very bright in ${ }^{12} \mathrm{CO} J=6-5$, notably at distances less than $\sim 2$ " from the equator. ${ }^{12} \mathrm{CO} J=3-2$ is about a factor two weaker in these regions, and ${ }^{13} \mathrm{CO} J=3-2$ is much weaker, almost undetected. This shows that the observed lines are not opaque in the outflow and that its temperature is relatively high $(\gtrsim 100 \mathrm{~K})$.

We performed a simple model fitting of our ${ }^{12} \mathrm{CO} J=$ 3-2 maps. We used a code that is very similar to the one described by Bujarrabal et al. (2005). In this code, the excitation is described by a local thermodynamic equilibrum (LTE) approximation; LTE can be assumed for low- $J$, high-opacity transitions, which are easily thermalized (Paper I), but not in general. 
V. Bujarrabal et al.: ALMA observations of the Red Rectangle, a preliminary analysis

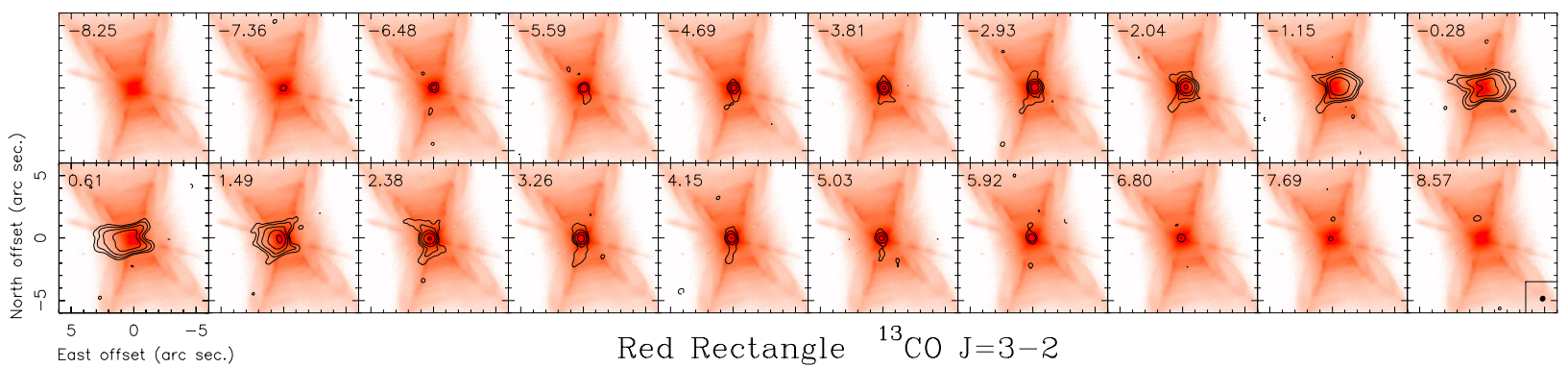

Fig. 2. Same as Fig. 1 for ${ }^{13} \mathrm{CO} J=3-2$. For this weak line, the continuum has been subtracted and the spectral resolution has been degraded to $0.84 \mathrm{~km} \mathrm{~s}^{-1}$. The contour spacing is logarithmic: $\pm 0.015,0.045,0.135,0.405$, and $1.215 \mathrm{Jy} /$ beam $( \pm 0.51,1.53,4.59,13.8$, and $41.3 \mathrm{~K})$.

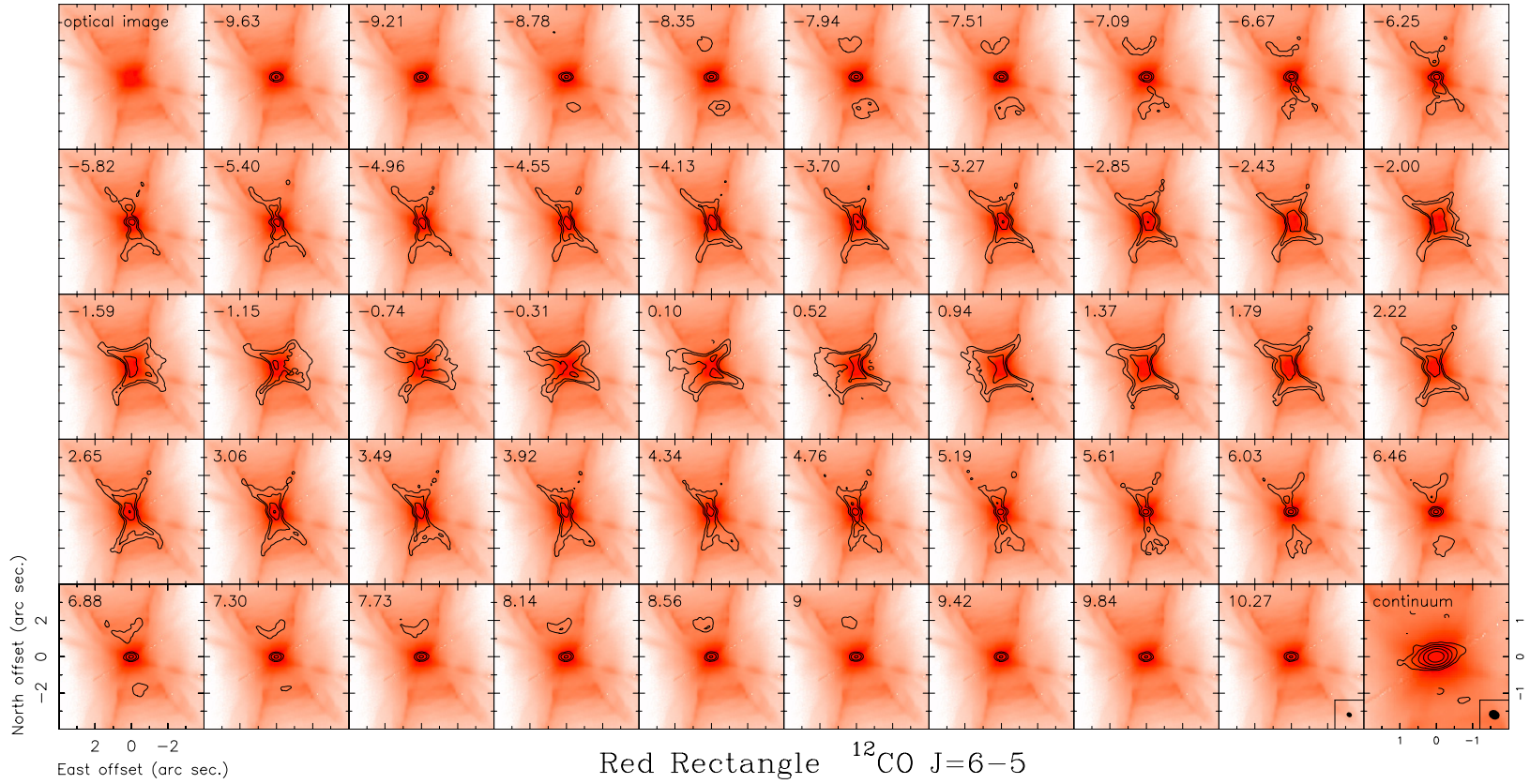

Fig. 3. Same as Fig. 1 but for ${ }^{12} \mathrm{CO} J=6-5$. The contour spacing is logarithmic: $-0.3,0.2,0.6,1.8$, and 5.4 Jy/beam (6.7, 20.0, 60.1, and 180.4 K). $0.45 \mathrm{~mm}$ continuum contours are $\pm 0.01,0.03,0.09,0.27$, and $0.81 \mathrm{Jy} /$ beam.

For that reason we applied this treatment only to ${ }^{12} \mathrm{CO} J=3-2$, and here we just give a brief discussion of the modeling of our ALMA results. A more detailed treatment of the line excitation, including model fitting for all lines, requires complex calculations (using an "exact" non-local treatment in two dimensions, Paper I) and a very careful and detailed discussion of the results, which is clearly beyond the scope of this Letter, so it is delayed to a forthcoming paper.

We adopted the same disk model deduced in Paper I (where all parameters are discussed in detail). We only decreased the disk density by a factor of 2 , because the predicted line emission from this component was stronger than observed. We introduced a new component in expansion, which is kept as simple as possible and symmetric with respect to the polar axis and equatorial plane. The results of our modeling, in the form of synthetic maps of ${ }^{12} \mathrm{CO} J=3-2$ in the same units as the observational data, are shown in Fig. 4. The shape, dynamics, and density in the bestfit model nebula are depicted in Fig. 5; the temperature of the outflow is assumed to be constant and equal to $200 \mathrm{~K}$. In Fig. 5 , we represent parameters in a plane perpendicular to the equator, therefore the disk is seen edge on and the rotational velocity, which is the same as in Paper I, cannot be displayed.

As we see, the model prediction is satisfactory. The emission of the disk is reproduced well when assuming practically the same structure and conditions as those derived in Bujarrabal et al. (2005) and Paper I. The emission from the expanding gas (at relatively high velocity shifts) is also explained well. It is remarkable that the properties of the outflow are roughly compatible with those tentatively deduced in Paper I to explain the profiles of high- $J$ CO lines. However, several caveats must be kept in mind. First of all, the nebula is not exactly symmetric with respect to the equator and the axis. This cannot be reproduced by our model, which tends to give a kind of average. Second, we assumed a somewhat lower disk density than previously thought. This may result from the contribution of the outflow to the total emission and our LTE assumption, which would not account for some underexcitation in this line (since the density is not very high). We recall that a more detailed modeling must include the fitting of our ${ }^{13} \mathrm{CO} J=3-2$ and ${ }^{12} \mathrm{CO} J=6-5$ maps and much more complex and difficult calculations, so is deferred to a forthcoming work. Our modeling is preliminary, but we think that it yields the main results that can be extracted from our data and offers a reasonable description of the nebula.

\section{Conclusions}

We present high-quality ALMA observations of ${ }^{12} \mathrm{CO}$ and ${ }^{13} \mathrm{CO} J=3-2$, and ${ }^{12} \mathrm{CO} J=6-5$ line emission in the Red Rectangle, see Figs. 1-3. In these figures we also show the maps of the submm-wave continuum, as well as an HST image of the nebula for comparison (Sect. 2). We performed preliminary 


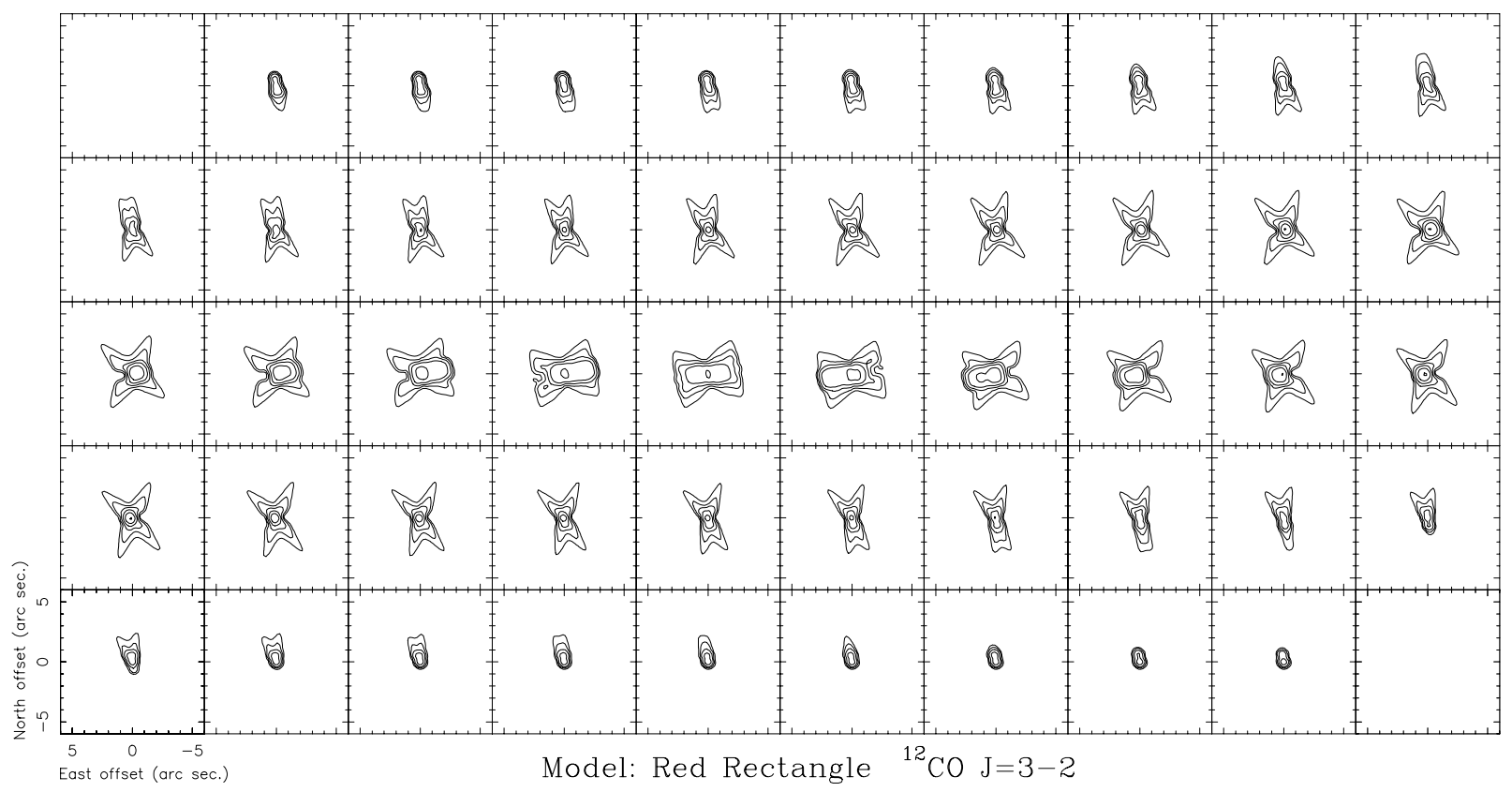

Fig. 4. Calculations from our best-fit simple model for the ${ }^{12} \mathrm{CO} J=3-2$ line emission. The contours and angular and velocity units are the same as for Fig. 1, which shows the observational data.

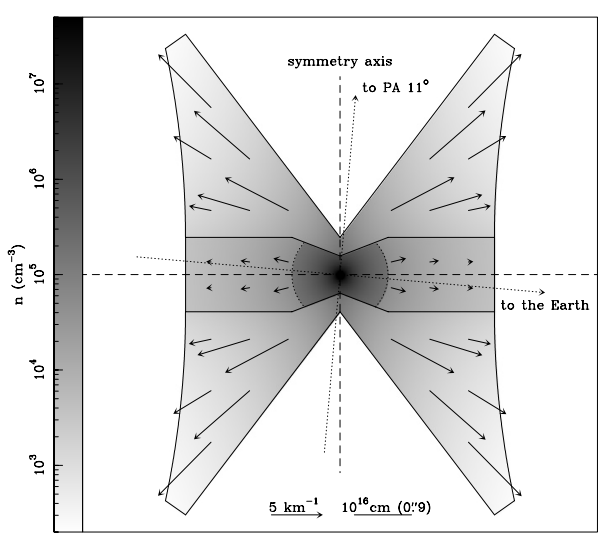

Fig. 5. Structure, velocity, and density distribution in our best-fit model disk and outflow. We represent parameters for a plane perpendicular to the equator; i.e., the disk is seen edge-on and only expansion is shown.

modeling of the ${ }^{12} \mathrm{CO} J=3-2$ line (which is the easiest to model, Sect. 3), which yielded satisfactory predictions. Our bestfit synthetic maps and deduced nebula properties are shown in Figs. 4 and 5. Our main results can be summarized as follows.

1: The Red Rectangle is a complex nebula, showing both a rotating disk and a component in expansion. The coexistence of extended rotating disks and outflows is probably common in post-AGB objects, at least in low-mass nebulae surrounding binary stars (Sect. 1, Paper II).

2: The high brightness measured in the central panels suggests opaque lines in the disk. ${ }^{12} \mathrm{CO} J=6-5$ emission is particularly intense in the outflow, while ${ }^{13} \mathrm{CO} J=3-2$ is weak, indicating relatively high excitation and low opacities in this component.

3: Our interpretation of the data is compatible with the properties of the disk deduced in previous works.
4: The gas in expansion basically occupies the region placed within the disk and the double cone delineated by the $\mathrm{X}$-shaped optical images. The optical nebula must then correspond to the inner illuminated region of the expanding lobes and the density within the double cone would be much lower. The velocity of the outflow is moderate, $\sim 10 \mathrm{~km} \mathrm{~s}^{-1}$, with a trend toward decreasing in regions close to the equator. The density of this expanding component increases toward the center, with values between $10^{3}$ and $10^{5} \mathrm{~cm}^{-3}$, and the temperature of the gas is kept constant at $200 \mathrm{~K}$.

Acknowledgements. This work has been supported by the Spanish MICINN, program CONSOLIDER INGENIO 2010, grant "ASTROMOL" (CSD200900038). CSC is partially supported by the Spanish MINECO, grants AYA2009-07304 and AYA2012-32032. We used the SIMBAD and MAST databases. This paper makes use of the following ALMA data: ADS/JAO.ALMA\#2011.0.00223.S. ALMA is a partnership of ESO (representing its member states), NSF (USA) and NINS (Japan), together with NRC (Canada) and NSC and ASIAA (Taiwan), in cooperation with the Republic of Chile. The Joint ALMA Observatory is operated by ESO, AUI/NRAO, and NAOJ.

\section{References}

Balick, B., \& Frank, A. 2002, ARA\&A, 40, 439

Bujarrabal, V., \& Alcolea, J. 2013, A\&A, 552, A116 (Paper I)

Bujarrabal, V., Castro-Carrizo, A., Alcolea, J., \& Sánchez Contreras, C. 2001, A\&A, 377, 868

Bujarrabal, V., Castro-Carrizo, A., Alcolea, J., \& Neri, R. 2005, A\&A, 441, 1031

Bujarrabal, V., van Winckel, H., Neri, R., et al. 2007, A\&A, 468, L45

Bujarrabal, V., Alcolea, J., Van Winckel, H., et al. 2013, A\&A, in press, DOI: 10.1051/0004-6361/201322015 (Paper II)

Cohen, M., Van Winckel, H., Bond, H. E., \& Gull, T. R. 2004, AJ, 127, 2362

Frank, A., \& Blackman, E. G. 2004, ApJ, 614, 737

Men'shchikov, A. B., Schertl, D., Tuthill, P. G., et al. 2002, A\&A, 393, 867

Soker, N. 2001, ApJ, 558, 157

van Leeuwen, F. 2007, A\&A, 474, 653

Van Winckel, H. 2003, ARAA, 41, 391 


\section{Appendix A: Further details on observations and data reduction}

Two observing runs were carried out in band 7 on October 21 and 22, 2012, of 80 and $100 \mathrm{~min}$, respectively. With these we obtained a total of $71 \mathrm{~min}$ of correlations on source. The band 9 observations were performed in November 3 and 4, 2012, in four runs with durations of $133,127,64$, and $111 \mathrm{~min}$, which allowed accumulating a total of 135 min of correlations on source. For band 7, J0522-364 was observed for the RF calibration. For band 9, J0538-440 or 3 C84 were observed instead. Callisto was always observed for absolute flux calibration, but for one run in band 9 in which data in Ceres was obtained. J0607085 and J0609-157 were observed every 10 to 20 min for gain calibration.

ALMA staff provided first calibrated data in December 2012, which were considerably improved by us. In particular, we made sure that the absorption $\mathrm{CO}$ lines detected at the position of the $\mathrm{RF}$ calibrator did not get into source data. The bandpass was calibrated by fitting cubic splines. Also, having identified different RF solutions for the different observed runs, an independent calibration was performed per track. For gain calibration, data from the two observed calibrators were considered. Later, from these first calibrated data, self-calibration was carried out by using the bright and moderately extended continuum emission of the Red Rectangle. All those processes resulted in very significant improvement in the quality of final images.

The flux calibration was based on the Butler-JPL-Horizons 2010 model for solar system bodies. A recent upgrade in that model (including, e.g., Herschel data) suggests that the intensity presented here could be slightly overestimated, by $10 \%$ in band 7 and by $5-10 \%$ in band 9 .

In band 7 , the total observed frequency ranges were $330.354-330.822,333.152-333.620,345.106-345.574$, and $345.562-346.030 \mathrm{GHz}$, to map mainly the emission of the ${ }^{12} \mathrm{CO}$ and ${ }^{13} \mathrm{CO} J=3-2$ transitions. In band 9 , we observed the intervals 673.540-674.477, 677.040-677.977, 687.805-688.742, and $691.004-691.941 \mathrm{GHz}$ to image the ${ }^{12} \mathrm{CO} J=6-5$ line.

A WFPC2/f622w optical image was obtained from the HST archive. The optical and ALMA images were aligned by adopting the astrometry provided by the Hubble Legacy Archive, which we have checked is reliable using the 2MASS coordinates of the 11 field stars detected within the WFPC2 field, and correcting for the Red Rectangle's proper motion (as measured by HIPPARCOS and listed in the SIMBAD database, $\delta_{\mathrm{RA}}=$

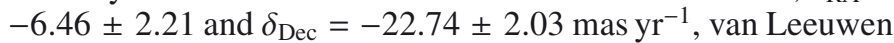
2007) for a time difference of $13.3 \mathrm{yr}$ between the HST and ALMA observations. The resulting alignment of the centroids, within $\$ 00^{\prime} \cdot 1$, is found to be satisfactory, in view of the uncertainty in the stellar positions and proper movements. 